PII: S0038-1098(97)10109-0

\title{
ANISOTROPIC TUNNELING IN InGaAsP/InP MULTI-QUANTUM BARRIER STRUCTURE
}

\author{
Fabio Liaci, ${ }^{a}$ Donato Greco, ${ }^{a}$ Roberto Cingolani, ${ }^{a}$ Domenico Campi, ${ }^{b}$ Cesare Rigo ${ }^{b}$ and Domenico Soldani ${ }^{b}$ \\ ${ }^{a}$ Dipartimento di Scienza dei Materiali, Università di Lecce, Via per Arnesano, I-73100 Lecce, Italy \\ ${ }^{b}$ Centro Studi e Laboratori Telecomunicazioni (CSELT), Via G. Reiss Romoli 274 I-10148 Torino, Italy
}

\begin{abstract}
(Received 23 November 1996; accepted 2 September 1997 by E. Molinari)
\end{abstract}
\begin{abstract}
We present magneto-photoluminescence experiments on a multi-quantumbarrier structure consisting of 31 period of wide $(58 \mathrm{~nm})$ quaternary wells of InGaAsP with the same lattice parameter as InP separated by thin $(8 \mathrm{~nm})$ InP barriers. The anomalous diamagnetic shift and the asymmetric broadening of the luminescence spectra evidence strong band filling in the tail of the density of states of the quaternary alloy, suggesting that tunneling transport through the $\mathrm{InP}$ barrier is anisotropic along the growth axis, due to different band lineups for the direct (InGaAsP-on-InP) and inverse (InP-on-InGaAsP) interfaces. This finding is supported by high resolution $\mathrm{X}$-ray patterns, yielding evidence that the direct and inverse interfaces are smooth and display different chemical compositions. (C) 1997 Elsevier Science Ltd
\end{abstract}

The InGaAs(P)/nP system is of particular relevance to optoelectronic devices operating in the wavelength range $1.3-1.5 \mu \mathrm{m}$, where minimum-loss of commercial optical fibers occurs. In this communication we report on the observation of band filling in the tail of the density of states of quaternary $(Q)$ InGaAsP quantum wells under the action of a transverse magnetic field. The observed anomalous behavior of the diamagnetic shift and asymmetric broadening of the magnetoluminescence spectra can be explained quantitatively by assuming different band lineups for the direct (Q-on-InP) and inverse (InP-on-Q) interfaces in our structures. This finding is strongly supported by highresolution X-ray diffraction (HRXRD) experiments, demonstrating the different nature of the direct and inverse interfaces, which causes different tunneling probabilities depending on the direction of carriers crossing the interfaces ("left" to "right" or the opposite one).

The semiconductor heterostructure used in this study was grown by Chemical Beam Epitaxy and nominally consisted of a stack of thirty-one, $58 \mathrm{~nm}$ thick InGaAsP layers, separated by $8.0 \mathrm{~nm}$ InP barriers. Below this region there was a $0.3 \mu \mathrm{m}$ InP buffer layer and above it a $0.2 \mu \mathrm{m}$ capping layer. The material in the structures was nominally undoped and unstrained. Room-temperature and low-temperature photoluminescence (PL) peaks were found at $0.89 \mathrm{eV}$ and $0.95 \mathrm{eV}$, respectively. The sample was grown at a substrate temperature of $515^{\circ} \mathrm{C}$, using group V element/group III element flux ratio of 40 (InP) and 20 (Q) corresponding to growth rates of $0.38 \mu \mathrm{m} \mathrm{h}^{-1}$ (InP) and $0.53 \mu \mathrm{m} \mathrm{h}^{-1}$ (Q). At each interface, the growth was stopped to allow planarization and atomic readjustment: the interruption time was set to $5 \mathrm{~s}$ and $10 \mathrm{~s}$ for the Q-on-InP interface and for the InP-on-Q interface, respectively. We note further that the switching-time of the hydride gas sources was set to $1 \mathrm{~s}$ to switch from phosphine to phosphine plus arsine at the Q-on-InP interface and $10 \mathrm{~s}$ for the reverse process at the InP-on-Q interface. Careful luminescence (PL) and magneto-luminescence (MPL) experiments were conducted at liquid helium temperature (around $3 \mathrm{~K}$ ) in order to study tunneling transport without thermal hopping contribution. The field was applied along the growth axis, with a flux up to $8 \mathrm{~T}$. PL was performed in the backscattering configuration and the excitation was provided by the green line of an $\mathrm{Ar}^{+}$laser, with typical excitation density of $20 \mathrm{~W} \mathrm{~cm} \mathrm{~cm}^{-2}$. The collected light was dispersed in a Jobin-Yvon monochromator with $1 \mathrm{~m}$ focal length. The signal was detected by a Ge-photodiode cooled at $77 \mathrm{~K}$ with liquid nitrogen and using standard lock-in techniques.

Typical MPL spectra are displayed in Fig. 1. With increasing magnetic field, the integrated emission 


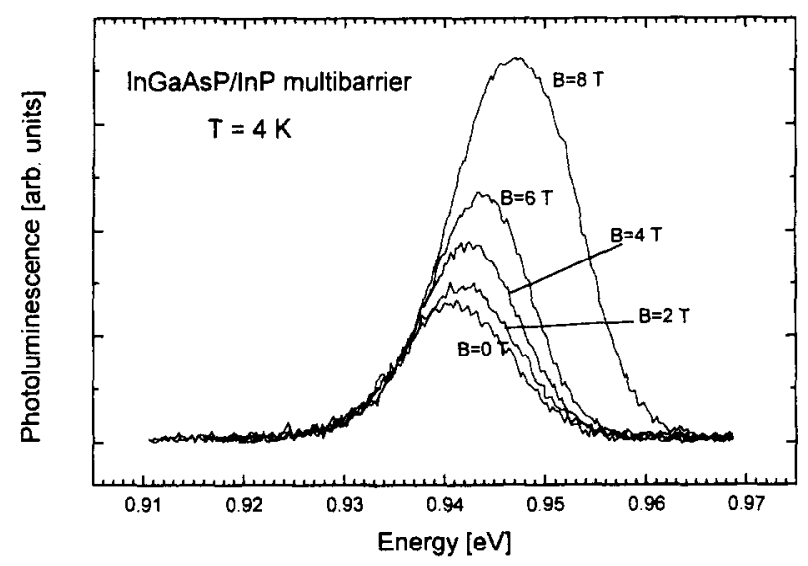

Fig. 1. Magnetoluminescence spectra of the InGaAsP/InP quantum wells at different magnetic flux values. The temperature is $4 \mathrm{~K}$.

intensity monotonically increases and the emission bandwidth broadens considerably towards high energies. Furthermore, the peak energy undergoes a blue-shift reaching a maximum value of $5.8 \mathrm{meV}$ at 8 Tesla. Emission enhancement and blue-shift (diamagnetic shift) are usual features in the MPL of semiconductors, since the applied field induces a localization of the carrier wavefunctions, resulting in the enhancement of the oscillator strength and emission intensity. However, the strong asymmetric broadening of the MPL spectra is quite unexpected (spin splitting of the band-edges in nonmagnetic semiconductors is very weak - less than $1 \mathrm{meV}$ - and should not be resolved within the inhomogeneous broadening of the PL line). In addition, the measured blue-shift of the MPL spectra does not match either the excitonic diamagnetic shift (proportional to $B^{2}$ ) or the free-carrier Landau shift (linear in $B$ ). It was clear that the observed blue-shift displays a dependence vs the magnetic field of degree higher than two. Consequently the results of Fig. 1 reveal the occurrence of some additional physical phenomenon, which dominates the blue-shift of the maximum emission energy and grows faster than $B^{2}$. Actually, we believe that we observed the band filling of the recombining electron states under the action of the applied magnetic field. We show here that band filling qualitatively accounts for the three features reported above, including the broadening of the peak. Moreover, comparison of the PL with absorption spectra suggests that the filled states pertain to the band tail resulting from compositional fluctuations within the alloy material. Filling of localized states can also be inferred from Fig. 1, showing that the low-energy wing of the PL spectra is not modified by the external magnetic field.

Let us recall that the manifestation of band filling was firstly observed in forward-biased $p-n$ homojunctions during the 1960s [1]. There, band-filling was a special case of photon-assisted tunneling through a junction in a bulk semiconductor, for biases just below the injection regime. However, the considerations on the emission characteristics in [1] apply to our case as well, since these do not depend on the filling mechanism itself. In particular, assuming a distribution of tail states proportional to $\exp \left(E / E_{0}\right)$, the shape of the low-energy edge of the PL should be proportional to $\exp \left(h v / E_{0}\right)$ and the integrated PL intensity $I$ should follow

$I=I_{0} \exp \left(\frac{h \nu_{m}}{E_{0}}\right)$

where $h v_{m}$ is the energy of the emission maximum.

Figure 2 displays our experimental points, together with the best-fit using equation (1). For $B \geq 2 \mathrm{~T}$ the results are in excellent agreement with the theory. The best-fit value we obtain for $E_{0}$ is $5.18 \pm 0.15 \mathrm{meV}$. We also checked the shape of the low-energy edge of the PL, with the striking result that the exponential behavior was correctly described using $E_{0}=5.05 \pm 0.1 \mathrm{meV}$, that is, the same value as above within the error bars. Based on these results we can explain the observed band filling according to the following considerations.

The difference between the electron effective masses of the InP barrier and the InGaAsP well results in different Landau shifts of the conduction band. Since the mass is heavier in the barrier than in the well, the overall result is a reduction of the height of the potential barrier experienced by the electron, which in turn causes the enhancement of the tunneling with increasing $B$. The opposite happens for the holes, whose masses are comparable (heavy-holes) or even lighter (light-holes) in the barrier. In this case tunneling is inhibited by the magnetic field. However at this point nothing should be

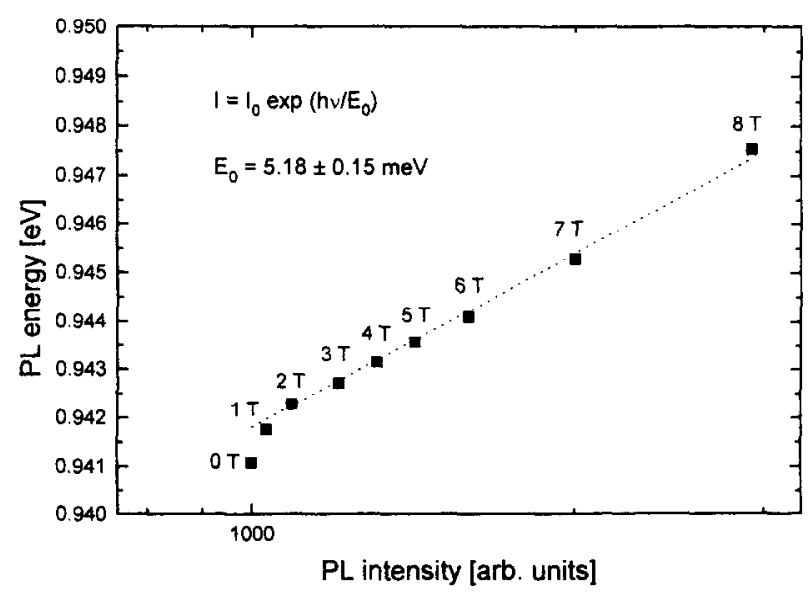

Fig. 2. Peak energy vs integrated emission intensity of the magneto-luminescence measured at different magnetic filed values (symbols). The dashed line represents the best fit with the band-tail filling model (see text). 
observable, since the net current through a barrier is zero because of identical probability for tunneling from the "left" and "right" and vice-versa. We thus expect that the actual effective band lineups has to be different for the direct (Q-on-InP) and the inverse (InP-on-Q) interfaces, leading to different barrier heights depending on the direction of motion of the tunneling carriers and resulting in a finite net current through the barriers. This current would be large enough to fill the tail states near the barrier. The above discussion indicates electrons as the filling carriers, but the opposite hehavior of the holes (tunneling away from the accumulation region) should in principle contribute to the phenomenon by decreasing the recombination rate. A calculation - performed assuming symmetrical band lineups and that $60 \%$ of the band discontinuity lies in the valence band - yields values of $7 \mathrm{ps}$ for electrons and $3 \mathrm{~s}$ and $28 \mathrm{ps}$ for heavy- and light-holes, respectively. This provides us a good reason for holding electrons as the filling carriers and for discarding the role of the holes. The physical picture emerging from these considerations explains the driving role of the applied fields, since larger Landau shifts promoted by increased B values, induce electrontunneling probabilities from the low-barrier side to the high-barrier side, that are increasingly larger as compared to the opposite process.

The occurrence of asymmetric band lineups in our heterostructures is very likely, since the two materials share neither common cationic (In becomes InGa) nor common anionic (P becomes AsP) sublattices [2]. In such cases, the interfacial bond lengths display strong differences depending on the particular interfacial structure [3], resulting into strained bonds and dipoles which alterate the band lineups [4] for growth on polar surfaces such as $\left(\begin{array}{lll}0 & 0 & 1\end{array}\right)$ or $\left(\begin{array}{ll}1 & 1\end{array}\right)$, even if the bulk materials are lattice-matched. Therefore, a difference in the interface structure as a function of the order of growth can lead to different interface strains and thus to non-commutative band lineups. We do not discuss here the parameters that may produce such differences in the interface configurations and refer to the literature cited; we just mention the example of In segregation in InGaAs alloys [5]. In order to assess if our structure actually displays such interface asymmetry, we performed HRXRD measurements.

In Fig. 3 we show the experimental and theoretical HRXRD patterns around the $(004)$ reflection of our sample. The measurements were carried out on a $X$-PERT Philips diffractometer with a beam divergence of twelve arc seconds, investigating a small sample area of $500 \mu \mathrm{m} \times 500 \mu \mathrm{m}$, in order to reduce possible contributions from lateral inhomogenities. The calculation of the model rocking curve was carried out, based on the dynamical theory of diffraction. A

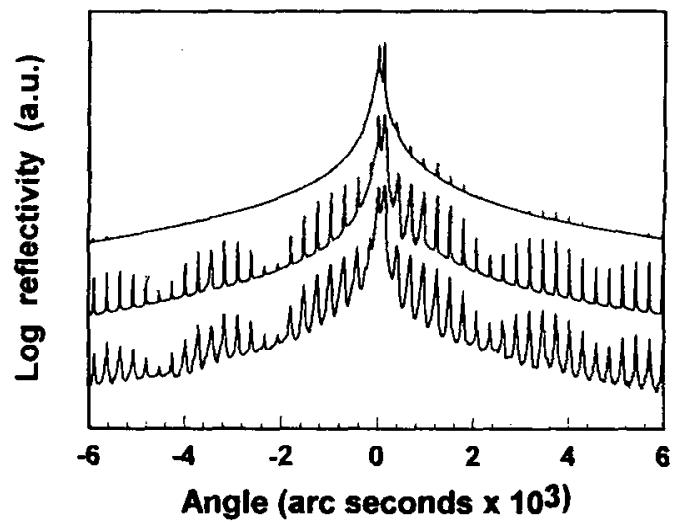

Fig. 3. High resolution $X$-ray diffraction curves of the investigated quaternary alloy quantum well structure. The bottom trace is the measured one. The middle trace is the calculated diffraction pattern, accounting for the existence of extended interfaces, whose thickness and compositions were determined by fitting the model curve to the experimental data. The topmost trace is the model curve fitted to the experimental data assuming abrupt and equivalent interfaces.

good overall agreement between the experimental and theoretical patterns could be obtained only by assuming the presence, in the structure period, of four layers having different chemical compositions and thicknesses and corresponding to the presence of extended, smooth interfaces. The fitting of the calculated HRXRD pattern to the measured one allowed the determination of compositions and thicknesses, being, respectively, $\mathrm{In}_{0.706} \mathrm{Ga}_{0.294} \mathrm{As}_{0.154} \mathrm{P}_{0.846}, 1.4 \mathrm{~nm}$ (Q-on-InP) and InAs ${ }_{0.252} \mathrm{P}_{0.748}, 2.0 \mathrm{~nm}$ (InP-on-Q). The composition and thickness of the $\mathrm{Q}$ layer resulting from the fitting were $\mathrm{In}_{0.606} \mathrm{Ga}_{0.394} \mathrm{As}_{0.834} \mathrm{P}_{0.166}$ and $58 \mathrm{~nm}$, while the thickness of the InP layers resulted $6.3 \mathrm{~nm}$. Clearly, the experimental and model outcomes provide a macroscopic average of a complicated chemical arrangement taking place at the interfaces on a microscopic scale. We can however consider that the two interfaces display different average compositions and, thus, undergo different interfacial strains, since the direct InGaP-like interface has a bulk lattice parameter about $1 \%$ smaller than that of InP, while the inverse InAsP-like one on the contrary displays a lattice parameter about $0.5 \%$ larger than InP. The asymmetrical interfacial strain is at the origin of different band line-ups for the direct and inverse interfaces. We stress here that such band lineups differences have already been experimentally observed in the InGaAs/InP system [6], with values for the direct/inverse valence band offset ratio as high as $170 \%$. Since our interfaces extend over more than $20 \AA$, a consequence is that narrow wells would be biased by the dipolar electric field. We note that we obtained evidence of this effect in the transmittance spectra of 
InGaAs/InP quantum wells of $5 \mathrm{~nm}$ nominal well width, displaying a very well resolved $e 1-h h 2$ structure, forbidden without electric field.

Finally, band lineups are expected to be slightly sensitive to temperature differences, because the magnitude of the thermal changes is comparatively smaller than the strain deformation at the interface bonds. Further, a very interesting feature of the lineup asymmetry would be that, for well chosen materials and interface configurations, the valence band (for example) could exhibit a type-I alignment with a staircase conduction band, making such structures attractive for realization of unipolar devices.

Acknowledgements-We gratefully acknowledge D. Cannoletta for expert technical help. One of us (F.L.) wishes to thank Dr P.C. Klipstein (Oxford) and Prof. M.H. Nazaré (Aveiro) for allowing him to spend some months in their Institutions.

\section{REFERENCES}

1. Pankove, J.I., Phys. Rev. Lett., 4, 1960, 20.

2. Foulon, Y., Priester, C., Allan, G., Garcia, J.C. and Landesman, J.P., J. Vac. Sci. Technol., B10, 1992, 1754.

3. When heterointerfaces share a common atom, the interface is well defined by the plane of common atom; therefore for an abrupt case, only a single interface configuration exists, leading to a single set of interfacial bond lengths; intermixing only spreads the band discontinuities over a larger distance (see [2]).

4. Hybertsen, M.S., Phys. Rev. Lett., 64, 1990, 555.

5. Moison, J.M., Guille, C., Houzay, F., Barthe, F. and Van Rompay, M., Phys. Rev., B40, 1989, 6149; Moison, J.M., Houzay, F., Barthe, F., Gérard, J.M., Jusserand, B., Massies, J. and Turco-Sandroff, F.S., J. Cryst. Growth, 111, 1991, 141.

6. Landesman, J.P., Garcia, J.C., Massies, J., Jezequel, G., Maurel, P., Hirtz, J.P. and Alnot, P., J. Vac. Sci. Technol., B10, 1992, 1761. 